\title{
ON THE $L_{q}$ NORM OF CYCLOTOMIC LITTLEWOOD POLYNOMIALS ON THE UNIT CIRCLE
}

\author{
TAMÁS ERDÉLYI
}

AbStract. Let $\mathcal{L}_{n}$ be the collection of all (Littlewood) polynomials of degree $n$ with coefficients in $\{-1,1\}$. In this paper we prove that if $\left(P_{2 \nu}\right)$ is a sequence of cyclotomic polynomials $P_{2 \nu} \in \mathcal{L}_{2 \nu}$, then

$$
M_{q}\left(P_{2 \nu}\right)>(2 \nu+1)^{a}
$$

for every $q>2$ with some $a=a(q)>1 / 2$ depending only on $q$, where

$$
M_{q}(P):=\left(\frac{1}{2 \pi} \int_{0}^{2 \pi}\left|P\left(e^{i t}\right)\right|^{q} d t\right)^{1 / q}, \quad q>0 .
$$

The case $q=4$ of the above result is due to P. Borwein, Choi, and Ferguson. A similar result is conjectured for Littlewood polynomials of odd degree. Our main tool here is the Borwein-Choi Factorization Theorem.

\section{INTRODUCTION}

Let $D$ be the open unit disk of the complex plane. Its boundary, the unit circle of the complex plane, is denoted by $\partial D$. Let $\mathcal{K}_{n}$ be the set of all polynomials of degree $n$ with complex coefficients of modulus 1 . Elements of $\mathcal{K}_{n}$ are often called (complex) unimodular polynomials of degree $n$. Let $\mathcal{L}_{n}$ be the set of all polynomials of degree $n$ with coefficients in $\{-1,1\}$. Elements of $\mathcal{L}_{n}$ are often called real unimodular polynomials or Littlewood polynomials of degree $n$. The Parseval formula yields

$$
\int_{0}^{2 \pi}\left|P_{n}\left(e^{i t}\right)\right|^{2} d t=2 \pi(n+1)
$$

for all $P_{n} \in \mathcal{K}_{n}$. Therefore

$$
\min _{z \in \partial D}\left|P_{n}(z)\right| \leq \sqrt{n+1} \leq \max _{z \in \partial D}\left|P_{n}(z)\right|
$$

An old problem (or rather an old theme) is the following.

2000 Mathematics Subject Classifications. 11C08, 41A17 
Problem 1.1 (Littlewood's Flatness Problem). How close can a $P_{n} \in \mathcal{K}_{n}$ or $P_{n} \in \mathcal{L}_{n}$ come to satisfying

$$
\left|P_{n}(z)\right|=\sqrt{n+1}, \quad z \in \partial D ?
$$

Obviously (1.1) is impossible if $n \geq 1$. So one must look for less than (1.1), but then there are various ways of seeking such an "approximate situation". One way is the following. In his paper [Li1] Littlewood had suggested that, conceivably, there might exist a sequence $\left(P_{n}\right)$ of polynomials $P_{n} \in \mathcal{K}_{n}$ (possibly even $P_{n} \in \mathcal{L}_{n}$ ) such that $(n+1)^{-1 / 2}\left|P_{n}\left(e^{i t}\right)\right|$ converge to 1 uniformly in $t \in \mathbb{R}$. We shall call such sequences of unimodular polynomials "ultraflat". More precisely, we give the following definition.

Definition 1.2. Given a positive number $\varepsilon$, we say that a polynomial $P_{n} \in \mathcal{K}_{n}$ is $\varepsilon$-flat if

$$
(1-\varepsilon) \sqrt{n+1} \leq\left|P_{n}(z)\right| \leq(1+\varepsilon) \sqrt{n+1}, \quad z \in \partial D .
$$

Definition 1.3. Given a sequence $\left(\varepsilon_{n_{k}}\right)$ of positive numbers tending to 0 , we say that a sequence $\left(P_{n_{k}}\right)$ of polynomials $P_{n_{k}} \in \mathcal{K}_{n_{k}}$ is $\left(\varepsilon_{n_{k}}\right)$-ultraflat if each $P_{n_{k}}$ is $\left(\varepsilon_{n_{k}}\right)$-flat. We simply say that a sequence $\left(P_{n_{k}}\right)$ of polynomials $P_{n_{k}} \in \mathcal{K}_{n_{k}}$ is ultraflat if it is $\left(\varepsilon_{n_{k}}\right)$-ultraflat with a suitable sequence $\left(\varepsilon_{n_{k}}\right)$ of positive numbers tending to 0 .

The existence of an ultraflat sequence of unimodular polynomials seemed very unlikely, in view of a 1957 conjecture of P. Erdős (Problem 22 in [Er]) asserting that, for all $P_{n} \in \mathcal{K}_{n}$ with $n \geq 1$,

$$
\max _{z \in \partial D}\left|P_{n}(z)\right| \geq(1+\varepsilon) \sqrt{n+1},
$$

where $\varepsilon>0$ is an absolute constant (independent of $n$ ). Yet, refining a method of Körner [Kö], Kahane [Ka] proved that there exists a sequence $\left(P_{n}\right)$ with $P_{n} \in \mathcal{K}_{n}$ which is $\left(\varepsilon_{n}\right)$ ultraflat, where

$$
\varepsilon_{n}=O\left(n^{-1 / 17} \sqrt{\log n}\right) .
$$

See also [QS]. A recent paper of Bombieri and Bourgain [BB] is devoted to the construction of ultraflat sequences of unimodular polynomials. In particular, one obtains a much improved estimate for the error term. A major part of this paper deals also with the long-standing problem of the effective construction of ultraflat sequences of unimodular polynomials.

Thus the Erdös conjecture (1.2) was disproved for the classes $\mathcal{K}_{n}$. For the more restricted class $\mathcal{L}_{n}$ the analogous Erdös conjecture is unsettled to this date. It is a common belief that the analogous Erdős conjecture for $\mathcal{L}_{n}$ is true, and consequently there is no ultraflat sequence of polynomials $P_{n} \in \mathcal{L}_{n}$. An interesting result related to Kahane's breakthrough is given in [Be]. For an account of some of the work done till the mid 1960's, see Littlewood's book [Li2] and [QS]. The structure of ultraflat sequences of unimodular polynomials is studied in [Er1], [Er2], [Er3], and [Er4], where several conjectures of Saffari are proved. 
The Rudin-Shapiro polynomials appear in Harold Shapiro's 1951 thesis at MIT and are sometimes called just Shapiro polynomials. See Chapter 4 of [Bo] for the construction(s). Cyclotomic properties of the Rudin-Shapiro polynomials are discussed in [BLM]. A sequence $\left(P_{n}\right)$ of Rudin-Shapiro polynomials satisfies $P_{n} \in \mathcal{L}_{n}$ and

$$
\left|P_{n}(z)\right| \leq C \sqrt{n+1}, \quad z \in \partial D,
$$

with an absolute constant $C$. In this paper we prove that a sequence of cyclotomic Littlewood polynomials of even degree is far from having the above "flatness" property of a sequence of Rudin-Shapiro polynomials. Note that (see page 271 of [BE], for instance) a Littlewood polynomial has Mahler measure one if and only if it is cyclotomic, that is, it has all its zeros on the unit circle $\partial D$.

For a polynomial $P$ let

$$
M_{q}(P):=\left(\frac{1}{2 \pi} \int_{0}^{2 \pi}\left|P\left(e^{i t}\right)\right|^{q} d t\right)^{1 / q}, \quad q>0,
$$

and

$$
M_{\infty}(P):=\max _{t \in[0,2 \pi]}\left|P\left(e^{i t}\right)\right|
$$

\section{Preliminary Results}

An unpublished observation of the author is the following.

Theorem 2.1. If $\left(P_{2 \nu}\right)$ is a sequence of cyclotomic polynomials $P_{2 \nu} \in \mathcal{L}_{2 \nu}$, then

$$
M_{\infty}\left(P_{2 \nu}\right)>(2 \nu+1)^{a}
$$

where $a:=1-\log _{3} \frac{\pi}{2}=0.5889 \ldots>\frac{1}{2}$.

The stronger result below is due to P. Borwein, Choi, and Ferguson [BCF].

Theorem 2.2. If $\left(P_{2 \nu}\right)$ is a sequence of cyclotomic polynomials $P_{2 \nu} \in \mathcal{L}_{2 \nu}$, then

$$
M_{4}\left(P_{2 \nu}\right)>(2 \nu+1)^{a}
$$

with $a=\frac{1}{4} \log _{2}(1+\sqrt{17})=0.5892 \ldots>1 / 2$.

In the proof of both theorems above the result of Borwein and Choi [BC] stated below has been a key.

Theorem 2.3. Every cyclotomic polynomial $P \in \mathcal{L}_{n}$ of even degree can be factorized as

$$
P(z)= \pm \Phi_{p_{1}}( \pm z) \Phi_{p_{2}}\left( \pm z^{p_{1}}\right) \cdots \Phi_{p_{r}}\left( \pm z^{p_{1} p_{2} \cdots p_{r-1}}\right),
$$

where $n+1=p_{1} p_{2} \cdots p_{r}$, the numbers $p_{j}$ are primes, not necessarily distinct, and

$$
\Phi_{p}(z)=\sum_{j=0}^{p-1} z^{j}=\frac{z^{p}-1}{z-1}
$$

is the p-th cyclotomic polynomial.

It is conjectured that this characterization also holds for polynomials $P \in \mathcal{L}_{n}$ of odd degree. This conjecture is based on substantial computation together with a number of special cases. 


\section{NeW Results}

Theorem 3.1. If $\left(P_{2 \nu}\right)$ is a sequence of cyclotomic polynomials $P_{2 \nu} \in \mathcal{L}_{2 \nu}$, then

$$
M_{q}\left(P_{2 \nu}\right)>(2 \nu+1)^{a}
$$

for every $q>2$ with some $a=a(q)>1 / 2$ depending only on $q$.

Theorem 3.2. If $\left(P_{2 \nu}\right)$ is a sequence of cyclotomic polynomials $P_{2 \nu} \in \mathcal{L}_{2 \nu}$, then

$$
M_{1}\left(P_{2 \nu}\right)<(2 \nu+1)^{b}
$$

with some absolute constant $0<b<1 / 2$.

It is conjectured that similar results hold for cyclotomic Littlewood polynomials of odd degree.

\section{ProOFs}

Although Theorem 2.2 beats Theorem 2.1, we present the short proof of Theorem 2.1 that is simpler than and quite different from that of Theorem 2.2.

Proof of Theorem 2.1. We use the factorization theorem of Borwein and Choi. We prove the theorem by induction on the number of factors. The theorem is obviously true when $P_{2 \nu}$ has only one factor. The proof of the inductive step goes as follows.

Suppose the theorem is true for $f$, where $f$ has $k-1$ factors. We have to prove that theorem is true for

$$
g(z):=\Phi_{p}( \pm z) f\left(z^{p}\right) .
$$

Let $M(f)$ be the maximum modulus of $f$ on the unit circle. The key observation is that $M(f)$ is achieved by $\left|f\left(z^{p}\right)\right|$ at a system of $p$ equidistant points on the unit circle. Denote these by $z_{1}, z_{2}, \ldots, z_{p}$. Then there is at least one $z_{j}$ such that the angular distance between 1 and $z_{j}$ is at most $2 \pi /(2 p)$. Similarly there is at least one $z_{j}$ such that the angular distance between -1 and $z_{j}$ is at most $2 \pi /(2 p)$. Now the proof can be finished by Lemma 4.1 below the proof of which is a straightforward geometric argument. Using Lemma 4.1 the proof of the inductive step is obvious, since $a:=1-\log _{3} \frac{\pi}{2}$ ensures $(2 / \pi) p \geq p^{a}$ for every $p \geq 3$. In fact, using the prime factorization of $2 \nu+1$, where $2 \nu$ is the degree of $P_{2 \nu}$, one can get a larger value of the exponent $a$ in the theorem if the primes in the factorization of $2 \nu+1$ are large.

Lemma 4.1. If $z$ is a point on the unit circle such that the angular distance of $z$ from 1 is at most $2 \pi /(2 p)$, then $\left|\Phi_{p}(z)\right| \geq(2 / \pi) p$. If $z$ is a point on the unit circle such that the angular distance of $z$ from -1 is at most $2 \pi /(2 p)$. Then $\left|\Phi_{p}(-z)\right| \geq(2 / \pi) p$.

Proof of Lemma 4.1. Recall that

$$
\Phi_{p}(z)=\frac{z^{p}-1}{z-1}
$$

and $|\sin t| \leq|t|$ for every $t \in \mathbb{R}$. 
To prove Theorem 3.2 we proceed as follows. First we introduce some notation. Associated with a positive integer $p$ and a function $f$ defined on the unit circle $\partial D$ of the complex plane let

$$
\begin{gathered}
g(t):=f\left(e^{i t}\right), \quad t \in \mathbb{R}, \\
f_{p}(z):=f\left(z^{p}\right) \Phi_{p}(z), \quad \Phi_{p}(z):=\sum_{j=0}^{p-1} z^{j}, \quad g_{p}(t):=f_{p}\left(e^{i t}\right),
\end{gathered}
$$

that is,

$$
g_{p}(t):=f\left(e^{i p t}\right) h_{p}(t), \quad h_{p}(t):=\Phi_{p}\left(e^{i t}\right) .
$$

Let

$$
I(f):=\int_{\partial D}|f(z)||d z|=\int_{0}^{2 \pi}\left|f\left(e^{i t}\right)\right| d t, \quad I(g):=\int_{0}^{2 \pi}|g(t)| d t,
$$

so $I(g)=I(f)$. The key to the proof of Theorem 3.2 is the following lemma that allows an induction on the number of factors in the decomposition of the cyclotomic polynomial $P_{2 \nu}$ given by Theorem 2.3 .

Lemma 4.2. If $f$ is a continuous function on $\partial D$ such that $|f(z)|=|f(\bar{z})|$ for every $z \in \partial D$, then

$$
I\left(f_{p}\right) \leq p^{\alpha} I(f)
$$

for every odd prime $p$ with an absolute constant $0<\alpha<1 / 2$.

Proof of Lemma 4.2. Let $k>0$ be an integer and we define

$$
L_{j, k, p}:=\int_{(j-1) \pi / k}^{j \pi / k}\left|g_{p}(t)\right| d t, \quad j=1,2, \ldots .
$$

Then

$$
L_{j, k, p}=L_{j+2 k, k, p}, \quad j=1,2, \ldots
$$

and

$$
\begin{aligned}
I\left(g_{p}\right) & =\int_{0}^{2 \pi}\left|g_{p}(t)\right| d t=2 \int_{0}^{\pi}\left|g_{p}(t)\right| d t=2 \sum_{j=1}^{k p} \int_{(j-1) \pi /(k p)}^{j \pi /(k p)}\left|g_{p}(t)\right| d t \\
& =2 \sum_{j=1}^{k p} \int_{(j-1) \pi /(k p)}^{j \pi /(k p)}\left|f\left(e^{i p t}\right)\right|\left|h_{p}(t)\right| d t=2 \sum_{j=1}^{k p}\left(\int_{(j-1) \pi /(k p)}^{j \pi /(k p)}\left|f\left(e^{i p t}\right)\right| d t\right) M_{j, k, p} \\
& =2 \sum_{j=1}^{k p}\left(\int_{(j-1) \pi / k}^{j \pi / k}\left|f\left(e^{i u}\right)\right| d u\right) \frac{1}{p} M_{j, k, p} \\
& =2 \sum_{\mu=1}^{k} L_{\mu, k, p} \frac{1}{p}\left(\sum_{\nu=0}^{(p-1) / 2} M_{\mu+2 \nu k, k, p}+\sum_{\nu=0}^{(p-3) / 2} M_{2 k+1-\mu+2 \nu k, k, p}\right) \\
& =2 \sum_{\mu=1}^{k} A_{\mu, k, p} L_{\mu, k, p},
\end{aligned}
$$


where

$$
M_{j, k, p}:=\max _{I_{j, k, p}}\left|h_{p}(t)\right|=\max _{I_{j, k, p}}\left|\Phi_{p}\left(e^{i t}\right)\right|
$$

with

$$
I_{j, k, p}:=\left[\frac{(j-1) \pi}{k p}, \frac{j \pi}{k p}\right], \quad j=1,2, \ldots, k p
$$

and

$$
A_{\mu, k, p}:=\frac{1}{p}\left(\sum_{\nu=0}^{(p-1) / 2} M_{\mu+2 \nu k, k, p}+\sum_{\nu=0}^{(p-3) / 2} M_{2 k+1-\mu+2 \nu k, k, p}\right), \quad \mu=1,2, \ldots, k
$$

Here we used that the assumptions on $f$ imply that the value of

$$
\int_{(\mu-1) \pi / k}^{\mu \pi / k}\left|f\left(e^{i u}\right)\right| d u
$$

remains the same when $\mu$ is replaced with $\mu+2 \nu k$ or $2 k+1-\mu+2 \nu k$. The proof of Lemma 4.2 now follows from Lemma 4.3.

Lemma 4.3. Let the numbers $A_{\mu, k, p}, \mu=1,2, \ldots, k$, be defined by (4.1). There is an absolute constant $0<b<1 / 2$ such that for every odd prime $p$ there is a positive integer $k$ such that

$$
A_{\mu, k, p} \leq p^{b}, \quad \mu=1,2, \ldots, k
$$

Proof of Lemma 4.3. It turns out that for large primes even $A_{\mu, 1, p} \leq c \log p$ is true, while for smaller primes we choose larger values of $k$ to establish (4.2). Observe that

$$
\left|\Phi_{p}\left(e^{i t}\right)\right|=\left|\frac{\sin (p t / 2)}{\sin (t / 2)}\right|
$$

hence

$$
\left|\Phi_{p}\left(e^{i t}\right)\right| \leq \frac{1}{\sin (t / 2)} \leq \frac{1}{\frac{2}{\pi} \frac{t}{2}}=\frac{\pi}{t}, \quad t \in(0, \pi]
$$

This implies

$$
\left|\Phi_{p}\left(e^{i t}\right)\right| \leq \min \left\{p, \frac{\pi}{t}\right\}, \quad t \in(0, \pi]
$$

Observe also that

$$
\left|\Phi_{p}\left(e^{i t}\right)\right| \leq \frac{1}{\sin (t / 2)} \leq \frac{1}{\frac{\sin (\pi / 4)}{\pi / 4} \frac{t}{2}} \leq \frac{\pi}{\sqrt{2} t}, \quad t \in(0, \pi / 2]
$$


hence

$$
\left|\Phi_{p}\left(e^{i t}\right)\right| \leq \min \left\{p, \frac{\pi}{\sqrt{2} t}\right\}, \quad t \in(0, \pi / 2] .
$$

If $\mu=k=1$, then using (4.3) and (4.4) we easily obtain

$$
\begin{aligned}
A_{1,1, p} & :=\frac{1}{p}\left(\sum_{\nu=0}^{(p-1) / 2} M_{1+2 \nu, 1, p}+\sum_{\nu=0}^{(p-1) / 2} M_{2+2 \nu, 1, p}\right)=\frac{1}{p} \sum_{j=1}^{p} M_{j, 1, p} \\
& \leq \frac{1}{p}\left(p+\sum_{j=1}^{(p-1) / 2-1} \frac{p}{\sqrt{2} j}+\sum_{j=(p-1) / 2}^{p-1} \frac{p}{j}\right) \\
& \leq 1+\sum_{j=1}^{(p-1) / 2-1} \frac{1}{\sqrt{2} j}+\sum_{j=(p-1) / 2} \frac{1}{j} \\
& \leq 1+\frac{1}{\sqrt{2}}+\frac{1}{\sqrt{2}} \int_{1}^{(p-1) / 2} \frac{d x}{x}+\frac{2}{p-1}+\int_{(p-1) / 2}^{p-1} \frac{d x}{x} \\
& =1+\frac{1}{\sqrt{2}}+\frac{1}{\sqrt{2}}(\ln (p-1)-\ln 2)+\frac{2}{p-1}+\ln 2 \\
& \leq 1+\frac{1}{\sqrt{2}}+\left(1-\frac{1}{\sqrt{2}}\right) \ln 2+\frac{2}{p-1}+\frac{1}{\sqrt{2}} \ln p \\
& \leq p^{0.48}
\end{aligned}
$$

for every prime $p \geq 23$. Here we used the fact that $\ln p / p^{0.48}$ is decreasing for $p \geq 23$. Hence the lemma holds for all primes $p \geq 23$ (we choose $k=1$ ). Further, the estimates

$$
\begin{aligned}
& A_{1,1,19} \leq 1+\frac{1}{\sqrt{2}} \sum_{j=1}^{8} \frac{1}{j}+\sum_{j=9}^{18} \frac{1}{j} \leq 3.7<\sqrt{19}, \\
& A_{1,1,17} \leq 1+\frac{1}{\sqrt{2}} \sum_{j=1}^{7} \frac{1}{j}+\sum_{j=8}^{16} \frac{1}{j} \leq 3.63<\sqrt{17}, \\
& A_{1,1,13} \leq 1+\frac{1}{\sqrt{2}} \sum_{j=1}^{5} \frac{1}{j}+\sum_{j=6}^{12} \frac{1}{j} \leq 3.42<\sqrt{13}
\end{aligned}
$$

and

$$
A_{1,1,11} \leq 1+\frac{1}{11 \sin (\pi / 22)}+\frac{1}{\sqrt{2}} \sum_{j=1}^{4} \frac{1}{j}+\sum_{j=5}^{10} \frac{1}{j} \leq 3.25<\sqrt{11},
$$

show that lemma holds for all primes $11 \leq p \leq 19$ (we choose $k=1$ ). 
Now we study the case $p=7$. We have

$$
\begin{gathered}
A_{1,4,7} \leq \frac{1}{7}(7+1+2.31+1.35+1.38+1.05+1.03) \leq 2.16<\sqrt{7}, \\
A_{2,4,7} \leq \frac{1}{7}(6.83+2.15+2.07+1.42+1.23+1.06+1.02) \leq 2.26<\sqrt{7}, \\
A_{3,4,7} \leq \frac{1}{7}(6.32+3.34+1.88+1.51+1.19+1.09+1.01) \leq 2.34<\sqrt{7},
\end{gathered}
$$

and

$$
A_{4,4,7} \leq \frac{1}{7}(5.52+4.50+1.73+1.61+1.15+1.11+1.01) \leq 2.38<\sqrt{7} .
$$

In the above four estimates we used that

$$
\begin{gathered}
M_{1,4,7}=7, \quad M_{2,4,7}=\frac{\sin (\pi / 8)}{\sin (\pi / 56)} \leq 6.83, \\
M_{3,4,7}=\frac{\sin (2 \pi / 8)}{\sin (2 \pi / 56)} \leq 6.32, \quad M_{4,4,7}=\frac{\sin (3 \pi / 8)}{\sin (3 \pi / 56)} \leq 5.52, \\
M_{5,4,7}=\frac{\sin (4 \pi / 8)}{\sin (4 \pi / 56)} \leq 4.50, \quad M_{6,4,7}=\frac{\sin (5 \pi / 8)}{\sin (5 \pi / 56)} \leq 3.34, \\
M_{7,4,7}=\frac{\sin (6 \pi / 8)}{\sin (6 \pi / 56)} \leq 2.15, \quad M_{8,4,7}=\frac{\sin (7 \pi / 8)}{\sin (7 \pi / 56)} \leq 1,
\end{gathered}
$$

and

$$
M_{j, 4,7}=\frac{1}{\sin ((j-1) \pi / 56)}, \quad j=9,10, \ldots, 28 .
$$

Hence the lemma is proved for $p=7$ (we choose $k=4$ ).

Now we study the case $p=5$. We have

$$
\begin{gathered}
A_{1,4,5} \leq \frac{1}{5}(5+0.74+0.59+0.42+0.40) \leq 1.43<\sqrt{5}, \\
A_{2,4,5} \leq \frac{1}{5}(4.88+1.56+1+0.80+0.72) \leq 1.80<\sqrt{5}, \\
A_{3,4,5} \leq \frac{1}{5}(4.53+2.42+1.25+1.09+0.93) \leq 2.05<\sqrt{5},
\end{gathered}
$$

and

$$
A_{4,4,5} \leq \frac{1}{7}(3.96+3.24+1.25+1.25+1) \leq 2.15<\sqrt{5},
$$

In the above four estimates we used that

$$
M_{1,4,5}=5, \quad M_{2,4,5}=\frac{\sin (\pi / 8)}{\sin (\pi / 40)} \leq 4.88,
$$




$$
\begin{gathered}
M_{3,4,5}=\frac{\sin (2 \pi / 8)}{\sin (2 \pi / 40)} \leq 4.53, \quad M_{4,4,5}=\frac{\sin (3 \pi / 8)}{\sin (3 \pi / 40)} \leq 3.96, \\
M_{5,4,5}=\frac{\sin (4 \pi / 8)}{\sin (4 \pi / 40)} \leq 3.24, \quad M_{6,4,5}=\frac{\sin (5 \pi / 8)}{\sin (5 \pi / 40)} \leq 2.42, \\
M_{7,4,5}=\frac{\sin (6 \pi / 8)}{\sin (6 \pi / 40)} \leq 1.56, \quad M_{8,4,5}=\frac{\sin (7 \pi / 8)}{\sin (7 \pi / 40)} \leq 0.74, \\
M_{9,4,5}=-\frac{\sin (9 \pi / 8)}{\sin (9 \pi / 40)} \leq 0.59, \quad M_{10,4,5}=\frac{\sin (10 \pi / 8)}{\sin (10 \pi / 40)}=1, \\
M_{11,4,5} \leq 1.25, \quad M_{12,4,5} \leq 1.25 \\
M_{13,4,5} \leq 1.25, \quad M_{14,4,5}=-\frac{\sin (13 \pi / 8)}{\sin (13 \pi / 40)} \leq 1.09 \\
M_{15,4,5}=-\frac{\sin (14 \pi / 8)}{\sin (14 \pi / 40)} \leq 0.80, \quad M_{16,4,5}=\frac{\sin (15 \pi / 8)}{\sin (15 \pi / 40)} \leq 0.42, \\
M_{17,4,5}=\frac{\sin (17 \pi / 8)}{\sin (17 \pi / 40)} \leq 0.40, \quad M_{18,4,5}=\frac{\sin (18 \pi / 8)}{\sin (18 \pi / 40)} \leq 0.72,
\end{gathered}
$$

and

$$
M_{19,4,5}=\frac{\sin (19 \pi / 8)}{\sin (19 \pi / 40)} \leq 0.93, \quad M_{20,4,5}=\frac{\sin (20 \pi / 8)}{\sin (20 \pi / 40)} \leq 1 .
$$

Hence the lemma holds for $p=5$ (we choose $k=4$ ).

Now we study the case $p=3$. We have

$$
\begin{gathered}
M_{1, k, 3}=3, \\
M_{j, k, 3}=1+2 \cos \frac{(j-1) \pi}{3 k}, \quad j=2,3, \ldots, 2 k,
\end{gathered}
$$

and

$$
M_{j, k, 3}=-\left(1+2 \cos \frac{j \pi}{3 k}\right), \quad j=2 k+1,2 k+2, \ldots, 3 k,
$$

hence with

$$
y:=\frac{(\mu-1) \pi}{3 k} \in[0, \pi / 3], \quad \mu=1,2, \ldots, k,
$$

we have

$$
\begin{aligned}
3 A_{\mu, k, 3} & =M_{\mu, k, 3}+M_{2 k+1-\mu, k, 3}+M_{2 k+\mu, k, 3} \\
& =\left(1+2 \cos \frac{(\mu-1) \pi}{3 k}\right)+\left(1+2 \cos \frac{(2 k-\mu) \pi}{3 k}\right)-\left(1+2 \cos \frac{(2 k+\mu) \pi}{3 k}\right) \\
& =1+2 \cos y+2 \cos \left(\frac{2 \pi}{3}-\frac{2 \pi}{3 k}-y\right)-2 \cos \left(\frac{2 \pi}{3}+\frac{2 \pi}{3 k}+y\right) \\
& =1+2(\cos y+\sqrt{3} \sin y)+c(k)=1+4 \sin \left(y+\frac{\pi}{6}\right)+c(k) \leq 5+c(k)
\end{aligned}
$$


with $c(k) \rightarrow 0$ as $k \rightarrow \infty$. Therefore

$$
A_{\mu, k, 3} \leq \frac{5}{3}+\frac{c(k)}{3}<\sqrt{3}, \quad k=1,2, \ldots, \mu,
$$

for all sufficiently large $k$. Thus the lemma is proved for $p=3$ (we choose a sufficiently large $k$ ).

Proof of Theorem 3.2. The polynomials $P_{2 \nu}$ can be factorized as it is given in Theorem 2.3. The theorem follows by induction on the number of factors in $P_{2 \nu}$. We use Lemma 4.2 in the inductive step.

Proof of Theorem 3.1. Let $f$ be a continuous function on $\partial D$ and let

$$
I_{q}(f):=M_{q}(f)^{q}=\frac{1}{2 \pi} \int_{0}^{2 \pi} \mid f\left(\left.e^{i t}\right|^{q} d t .\right.
$$

Then $h(q):=\log \left(I_{q}(f)\right)=q \log \left(M_{q}(f)\right)$ is a convex function of $q$ on $(0, \infty)$. This is a simple consequence of Hölder's inequality. For the sake of completeness we present the short proof of it. We need to see that

$$
I_{r}(f) \leq I_{p}(f)^{\frac{r-q}{p-q}} I_{q}(f)^{\frac{p-r}{p-q}},
$$

that is

$$
\left(\frac{1}{2 \pi} \int_{0}^{2 \pi} \mid f\left(\left.e^{i t}\right|^{r} d t\right)^{p-q} \leq\left(\frac{1}{2 \pi} \int_{0}^{2 \pi} \mid f\left(\left.e^{i t}\right|^{p} d t\right)^{r-q}\left(\frac{1}{2 \pi} \int_{0}^{2 \pi} \mid f\left(\left.e^{i t}\right|^{q} d t\right)^{q-r} .\right.\right.\right.
$$

To see this let

$$
\alpha:=\frac{p-q}{r-q}, \quad \beta:=\frac{p-q}{p-r}, \quad \gamma:=\frac{p}{\alpha}, \quad \delta:=\frac{q}{\beta},
$$

hence $1 / \alpha+1 / \beta=1$ and $\gamma+\delta=r$. Let

$$
F(t):=\left|f\left(e^{i t}\right)\right|^{\gamma}=\left|f\left(e^{i t}\right)\right|^{\frac{p(r-q)}{p-q}},
$$

and

$$
G(t):=\left|f\left(e^{i t}\right)\right|^{\delta}=\left|f\left(e^{i t}\right)\right|^{\frac{q(r-q)}{p-q}},
$$

Then by Hölder's inequality we conclude

$$
\frac{1}{2 \pi} \int_{0}^{2 \pi} F(t) G(t) d t \leq\left(\int_{0}^{2 \pi} F(t)^{\alpha} d t\right)^{1 / \alpha}\left(\int_{0}^{2 \pi} G(t)^{\beta} d t\right)^{1 / \beta}
$$

and (4.5) follows. Using the convexity of $\log \left(I_{q}\left(P_{2 \nu}\right)\right)$ on $(0, \infty)$, for $q>2$ we have

$$
I_{2}\left(P_{2 \nu}\right) \leq\left(I_{1}\left(P_{2 \nu}\right)\right)^{\frac{q-2}{q-1}}\left(I_{q}\left(P_{2 \nu}\right)\right)^{\frac{1}{q-1}}
$$

and from Theorem 3.2 we obtain

$$
2 \nu+1 \leq\left((2 \nu+1)^{b}\right)^{\frac{q-2}{q-1}}\left(I_{q}\left(P_{2 \nu}\right)\right)^{\frac{1}{q-1}},
$$

that is

$$
(2 \nu+1)^{(q-1)-(q-2) b} \leq I_{q}\left(P_{2 \nu}\right)=M_{q}\left(P_{2 \nu}\right)^{q},
$$

with an absolute constant $0<b<1 / 2$. Hence with $a=a(q):=(q-1-(q-2) b) / q$ we have

$$
(2 \nu+1)^{a}=(2 \nu+1)^{(q-1-(q-2) b) / q} \leq M_{q}\left(P_{2 \nu}\right) .
$$

Here $a=a(q):=(q-1-(q-2) b) / q>1 / 2$, since $(1-2 b)(q / 2-1)>0$. 


\section{REFERENCES}

[Be] J. Beck, "Flat" polynomials on the unit circle - note on a problem of Littlewood, Bull. London Math. Soc. (1991), 269-277.

[BN] E. Bombieri and J. Bourgain, On Kahane's ultraflat polynomials, J. Eur. Math. Soc. 11 (2009, 3), 627-703.

[Bo] P. Borwein, Computational Excursions in Analysis and Number Theory, Springer-Verlag, New York, 2002.

[BC] P. Borwein and K.S. Choi, On cyclotomic polynomials with \pm 1 coefficients, Experiment. Math. 8 (1995), 399-407.

[BCF] P. Borwein, K.S. Choi, and R. Ferguson, Norm of Littlewood cyclotomic polynomials, Math. Proc. Cambridge Philos. Soc. 138 (2005), 315-326.

[BE] P. Borwein and T. Erdélyi, Polynomials and Polynomial Inequalities, Springer-Verlag, New York, 1995.

[BLM] J. Brillhart, J.S. Lomont, and P. Morton, Cyclotomic properties of the Rudin-Shapiro polynomials, J. Reine Angew. Math. 288 (1976), 37-75.

[Er1] T. Erdélyi, The phase problem of ultraflat unimodular polynomials: the resolution of the conjecture of Saffari, Math. Ann. 321 (2001), 905-924.

[Er2] T. Erdélyi, How far is a sequence of ultraflat unimodular polynomials from being conjugate reciprocal, Michigan J. Math. 49 (2001), 259-264.

[Er3] T. Erdélyi, A proof of Saffari's "near-orthogonality" conjecture for ultraflat sequences of unimodular polynomials, C. R. Acad. Sci. Paris Sér. I Math. 333 (2001), 623-628.

[Er4] T. Erdélyi, On the real part of ultraflat sequences of unimodular polynomials: consequences implied by the resolution of the Phase Problem, Math. Ann. 326 (2003), 489-498.

[Er] P. Erdős, Some unsolved problems, Michigan Math. J. 4 (1957), 291-300.

[Ka] J.P. Kahane, Sur les polynomes a coefficient unimodulaires, Bull. London Math. Soc. 12 (1980), 321-342.

[Kö] T. Körner, On a polynomial of J.S. Byrnes, Bull. London Math. Soc. 12 (1980), 219-224.

[Li1] J.E. Littlewood, On polynomials $\sum \pm z^{m}, \sum \exp \left(\alpha_{m} i\right) z^{m}, z=e^{i \theta}$, J. London Math. Soc. 41 (1966), 367-376.

[Li2] J.E. Littlewood, Some Problems in Real and Complex Analysis, Heath Mathematical Monographs, Lexington, Massachusetts, 1968.

[QS] H. Queffelec and B. Saffari, On Bernstein's inequality and Kahane's ultraflat polynomials, J. Fourier Anal. Appl. 2 (1996, 6), 519-582.

[Sa] B. Saffari, The phase behavior of ultraflat unimodular polynomials, in Probabilistic and Stochastic Methods in Analysis, with Applications (1992), Kluwer Academic Publishers, Printed in the Netherlands, 555-572.

Department of Mathematics, Texas A\&M University, College Station, Texas 77843

E-mail address: terdelyi@math.tamu.edu 